\title{
PSYCHOPHYSICS OF SUPPRESSION
}

\author{
RICHARD HARRAD \\ Bristol
}

\begin{abstract}
SUMMARY
In amblyopia the visual input from the amblyopic eye is suppressed. The difference in contrast sensitivity between the two eyes can lead to suppression of the amblyopic eye through dichoptic masking in anisometropic amblyopia and small angle strabismus. Binocular cells in the visual cortex may also be instrumental in bringing about suppression in small angle strabismus through fusional suppression and disparity-dependent suppression. In large angle strabismus without amblyopia suppression is very powerful and is probably a modified form of binocular rivalry suppression. Which particular mechanism is operating is dependent on the pathogenesis of the amblyopia, the depth of the amblyopia and, in strabismic amblyopia, the angle of strabismus. In any given subject more than one mechanism may be operating in the different areas of the field.
\end{abstract}

The child with strabismus and amblyopia does not suffer from double vision because the visual input of the deviating eye is suppressed. Von Graefe ${ }^{1}$ was the first to demonstrate a suppression scotoma in an amblyopic eye. He placed a red filter over the good eye and with the amblyopic eye fixing a light in the centre of a screen performed perimetry in the usual way. The presence of a suppression scotoma in the amblyopic eye was indicated by the test object turning red. Travers ${ }^{2}$ put a mirror in front of one eye and presented targets on separate screens to the two eyes independently. He found that the region of suppression in the deviating eye was in the area of the retina where the image presented at the fovea of the fixing eye falls, i.e. the diplopic point, and that the area of visual field suppressed was larger in dense amblyopia. In some patients Travers also found suppression at the fovea of the deviating eye (the point of confusion). These findings have been widely quoted and form the basis of many accounts of suppression in strabismus.

Jampolsky ${ }^{3}$ used prisms of increasing strengths

Correspondence to: R. Harrad, Bristol Eye Hospital, Lower Maudlin Street, Bristol BS1 2LX, UK. placed over one eye to measure the extent of the area of suppression. The subject was instructed to look at a distant light and the onset of diplopia was taken to indicate that the boundary of the scotoma had been reached. Like Travers he also found that the area of suppression included the point of confusion and the point of diplopia and that it was larger in dense amblyopia. However, he also found that in most patients he studied, the suppression scotoma stopped abruptly at the vertical meridian passing through the fovea. Jampolsky quotes his own experience and that of Posner and Schlossman ${ }^{4}$ that over-correction of a strabismus by as little as 2 or 3 prism dioptres will cause diplopia in most cases. $\mathrm{He}$ reported that there was an area of 'hemiretinal suppression' in the deviating eye which extended between the diplopic point and a vertical line running through the fovea. He made the assumption that the region where a target initiated suppression was the same as the area of suppression; however, this does not necessarily follow. He had located the area where suppression may be initiated but it is possible that the entire visual field of the deviating eye was suppressed when there was no diplopia. This is the conclusion reached by Pratt-Johnson and Tillson, ${ }^{5}$ who presented fusible targets on a modified Lees screen. They found that when strabismic patients were overcorrected with prisms, most developed diplopia, and they refer to the critical nature of the ocular alignment about the vertical midline as the hemiretinal trigger. They concluded that since diplopia was present throughout the binocular visual field after the midline had been transgressed, the entirety of this area must have been suppressed previously. This does not necessarily follow; it may be that diplopia is simply not perceived very readily in the periphery of the visual field. These authors and Sireteanu et al. ${ }^{6}$ found central suppression surrounded by peripheral fusion in microtropic subjects. Sireteanu and Fronius ${ }^{7}$ put forward an interesting argument to explain these findings. They suggest that the progressive enlargement of receptive field size from the fovea to the retinal periphery means that a

Eye (1996) 10, 270-273 c 1996 Royal College of Ophthalmologists 
small misalignment of the eyes will lead to loss of the capacity for constructive binocular interactions at the fovea where receptive field sizes are small, but that in the retinal periphery there will still be the possibility of facilitatory interactions and stereopsis since the physical separation of the cortical representation of these areas is very small. In order to abolish diplopia, suppression must therefore occur only in the central area around the fovea.

There are certain difficulties inherent in the study of suppression in strabismus. Firstly, in order to be able to distinguish between the stimuli presented to the two eyes they need to be different in some way. However, Jampolsky ${ }^{3}$ and Schor $^{8}$ have shown that similar stimuli are more likely to produce suppression than dissimilar stimuli, so any difference between the stimuli which enables the subject to distinguish them will tend to break down the suppression which is being measured. An analogy can be made here with Heisenberg's Uncertainty Principle. Workers who have used orthogonal patches of grating, or red and green stimuli viewed through red and green goggles, are likely to have underestimated the extent to which suppression occurs. In any given experiment the conclusion that is reached applies only to the immediate area of the visual field containing the suppressed stimulus; no firm conclusions can be drawn about what is happening elsewhere in the visual field where suppression may or may not be occurring. The above considerations go some way to explaining why the findings of different workers with regard to the area of suppression in strabismus show such differences.

Dichoptic masking is the physiological process where a stimulus of a given contrast presented to one eye can prevent the detection of a lower contrast but otherwise identical stimulus presented to the other eye. ${ }^{9,10}$ This binocular inhibitory process is known to be present in some amblyopic subjects. ${ }^{11}$ Harrad and Hess $^{12}$ put forward the following hypothesis: since there is a difference in the thresholds for contrast detection between the two eyes in amblyopic subjects, the normal eye will always receive input of a higher supra-threshold contrast and therefore the input to this eye should always mask the input to the amblyopic eye. If this is the case then dichoptic masking in the presence of a contrast threshold difference could account for suppression in amblyopia. They looked at a range of amblyopic subjects with different degrees of severity and types of amblyopia. They found that dichoptic masking could account for suppression in some anisometropic amblyopes and also mixed strabismic and anisometropic amblyopes at low spatial frequency once the difference in contrast threshold is accounted for. At high spatial frequencies in the mixed strab/aniso amblyopes there was no masking of the normal eye by the amblyopic eye although the amblyopic eye continued to be masked by the normal eye. In pure strabismic amblyopia with moderate amblyopia and in alternate fixation, suppression was more profound than would be expected on the basis of dichoptic masking alone. This is probably because dichoptic masking cannot operate once all retinal correspondence is lost. This loss of low contrast information due to dichoptic masking is similar to the process whereby a progressive reduction of the contrast of one of a pair of stereo half-images leads to the reduction of stereoacuity and eventually suppression of the half image having the lower contrast. ${ }^{13-15}$ It is probably this process, in the presence of reduced contrast sensitivity in anisometropic amblyopia, that is responsible for the poor stereoacuity in these patients. ${ }^{16}$ However, in subjects with deep strabismic amblyopia or alternate fixation, suppression cannot be accounted for by dichoptic masking.

Binocular rivalry occurs when each eye views two images that are so dissimilar that they cannot be fused. ${ }^{17-19}$ The observer experiences alternating dominance and suppression of each binocular image. This is a normal physiological process which is able to bring about perceptual suppression of the input of one eye. Harrad and Hess ${ }^{20}$ measured the threshold for the onset of rivalry between gratings presented independently to the two eyes which differed only in orientation. A grating of a given orientation was presented to one eye and a grating of a different orientation was presented to the other eye and the contrast of this second grating was increased from threshold until an episode of rivalry suppression occurred. They asked whether a raised threshold for binocular rivalry could account for suppression in amblyopia. They found that threshold elevation was seen for gratings of similar orientation, i.e. less than $30^{\circ}$ of orientation difference, in anisometropic amblyopia and in normal subjects. This was simply a measure of the orientational tuning of dichoptic masking. Threshold elevation also occurred for the amblyopic eye of mixed strabismic anisometropic subjects, while the normal eyes of these subjects showed a very small threshold elevation with no orientational tuning. These findings supported the view that the stimuli which are most likely to produce suppression, i.e. in the case of gratings those of similar orientation, are not those which produce binocular rivalry. The similarity between the orientational tuning in anisometropic amblyopia, mixed strabismic and anisometropic amblyopia and in normal subjects suggests that a form of orientational tuned contrast masking is producing suppression in these patients. One alternately fixating amblyopic subject showed a very high threshold elevation in either eye in the absence of any orientational tuning. 
To summarise, Harrad and $\mathrm{Hess}^{20}$ found that the suppression was relatively weak and orientationally tuned in anisometropic amblyopia, more powerful but still orientationally tuned in the presence of strabismus, and very powerful and lacking in orientational tuning in alternate fixation. The lack of orientational tuning in alternate fixation is to be expected from the results of animal experiments which show that such subjects are likely to have very few binocularly driven visual cortical cells. ${ }^{21}$ Blake $^{18}$ has proposed that powerful suppression seen in alternately fixating strabismics is necessary to inhibit the large monocular pool of cells driven by the contralateral eye. Holopigian et al. ${ }^{22}$ measured the depth of suppression in a group of amblyopic subjects and found an inverse correlation between the depth of suppression and the depth of amblyopia. Their results also show that suppression was most powerful in alternately fixating strabismics, intermediate in strabismic amblyopia and weakest of all in anisometropic amblyopia.

Many authors ${ }^{23,24}$ have suggested that binocular rivalry is the basis of suppression in amblyopia. However, there are a number of objections to this proposition: (i) the characteristic alternation of binocular rivalry is not seen in strabismic suppression; (ii) the wavelength suppression characteristics of binocular rivalry differ from those of strabismic suppression, ${ }^{25}$ in that normal observers exhibit wavelength-specific loss of sensitivity during the suppression phase of rivalry affecting the region between 400 and $470 \mathrm{~nm}$ but this wavelength-specific loss is not seen in strabismic suppression; (iii) the strength of suppression in strabismus has been found to be much stronger than rivalry suppression in normal subjects; ${ }^{21}$ and (iv) the visual stimuli, such as gratings of different orientation, that lead to binocular rivalry have tended to stimulate rivalry rather than suppression in amblyopia. ${ }^{8}$ However, it is probable that at the onset of strabismus in children over 6 months of age diplopia and binocular rivalry occur and it is likely that suppression in large angle strabismus and in those subjects that can alternately fix with either eye is a modified form of binocular rivalry.

Richards ${ }^{26}$ described stereoanomalous subjects who were unable to make judgements of depth for either crossed or uncrossed disparity. Schor ${ }^{8}$ showed that suppression was most pronounced for gratings of a similar orientation and that vertically orientated gratings provoked suppression more readily than horizontally orientated gratings, suggesting that horizontal disparities are more likely to be a stimulus for suppression than vertical ones. Schor ${ }^{27}$ measured the area of suppression in the deviating eye in terms of the position of targets presented to the fixating eye in a group of subjects with small angle strabismus. He found that suppression in his group of subjects was stimulated by a fixed disparity rather than suppression being confined to a particular area of the visual field. He found that uncrossed disparity led to suppression in his esotropic subjects and crossed disparity produced suppression in his exotropic subject. The subjects with normal retinal correspondence suppressed at zero disparity, while those with abnormal retinal correspondence suppressed at the abnormally corresponding point where the disparity was equal to the angle of the strabismus. There was therefore no evidence of selective suppression of all or part of one hemifield. He suggested that although suppression may be disparity dependent in small angle strabismus, this is unlikely to be the mechanism in large angle strabismus.

Observers with normal stereopsis suppress some of the information contained in each stereo half-imagea phenomenon called 'fusional suppression'. Where the two stereoscopic half-images consist of a vernier offset target presented to one eye and aligned vertical lines presented to the other eye, the observer is unable to make accurate positional judgements about the vernier component when the two halfimages are fused. McKee and $\mathrm{Harrad}^{28}$ predicted that since they lack a functional stereo system at the fovea, stereoanomalous observers would have better access to monocular positional information than normal observers. This prediction was confirmed; stereoanomalous observers were better at making real judgements for the stereo half-images than normal observers; the competition between units encoding conflicting information in the subjects seemed to be resolved by suppressing signals from the weaker eye. This fusional suppression was present for both vertical and horizontal disparities and for this reason it was felt to be a function of the fusion mechanism rather than the stereo mechanism. They suggested that the physiology amounted to suppression of small units coding for position by large units detecting large disparities, thus leading to loss of positional information.

It seems that there are a combination of processes operating to produce suppression in amblyopia. Which particular process is operating depends upon the pathogenesis of the amblyopia, the depth of amblyopia, the angle of the strabismus where present, and whether or not there is the capacity for alternate fixation. Dichoptic masking probably largely accounts for suppression in anisometropic amblyopia and in small angle strabismus. Disparityspecific suppression or fusional suppression may also be important in those patients with a small angle strabismus in the absence of amblyopia. In large angle strabismus where normal binocular interactions are not possible a powerful form of binocular rivalry suppression occurs. The differences between 
these processes in the normal population and in amblyopic subjects can be accounted for by modifications in the underlying neural circuitry which take place during visual development.

Key words: Amblyopia, Binocular rivalry, Strabismus, Suppression.

\section{REFERENCES}

1. von Graefe A. Das Sehen der Schielenden. Eine ophthalmologisch-physiologische Studie. Wiesbaden, 1896

2. Travers T. Suppression of vision in squint and its association with retinal correspondence and amblyopia. Br J Ophthalmol 1938;22:577-604.

3. Jampolsky A. Characteristics of suppression in strabismus. AMA Arch Ophthalmol 1955;54:683-96.

4. Posner A, Schlossman A. Relation of diplopia to binocular vision in concomitant strabismus. AMA Arch Ophthalmol 1951;45:615-22.

5. Pratt-Johnson JA, Tillson G. Suppression in strabismus: an update. Br J Ophthalmol 1984;68:174-8.

6. Sireteanu R, Fronius M, Singer W. Binocular interaction in the peripheral visual field of humans with strabismic and anisometropic amblyopia. Vision Res 1981;21:1065-74.

7. Sireteanu R, Fronius M. Naso-temporal asymmetries in human amblyopia: consequence of long-term interocular suppression. Vision Res 1981:21:1055-63.

8. Schor CM. Visual stimuli for strabismic suppression. Perception 1977;6:583-93.

9. Abadi RV. Induction masking: a study of some inhibitory interactions during dichoptic viewing. Vision Res 1976;16:269-75.

10. Legge GE. Spatial frequency masking in human vision: binocular interactions. J Opt Soc Am 1979;69:838-74.

11. Levi DM, Harwerth RS, Smith EL III. Humans deprived of normal binocular vision have binocular interactions tuned to size and orientation. Science 1979;206:852-4.

12. Harrad RA, Hess RF. Binocular integration of contrast information in amblyopia. Vision Res 1992;32:2135-50.

13. Halpern DL, Blake R. How contrast affects stereoacuity. Perception 1988;17:483-96.
14. Legge GE, Gu Y. Stereopsis and contrast. Vision Res 1989;29:989-1004.

15. Schor CM, Heckmann T. Interocular difference in contrast and spatial frequency: effects on stereopsis and fusion. Vision Res 1989;29:837-47.

16. Holopigian K, Blake R, Greenwald MJ. Selective losses in binocular vision in anisometropic amblyopia. Vision Res 1986;26:621-7.

17. Wheatstone C. Contributions to the physiology of vision. Part the first: On some remarkable, and hitherto unobserved, phenomena of binocular vision. Phil Trans R Soc Lond 1838;128:371-94.

18. Blake R. A neural theory of binocular rivalry. Psychol Rev 1989;96:145-67.

19. Fox R. Binocular rivalry. In: Regan D, editor. Vision and visual dysfunction, vol 9. London: Macmillan, 1991:93-110.

20. Harrad RA, Hess RF. A model for suppression in amblyopia. Transactions of the 20th Meeting of the European Strabismological Association 1992:113-8.

21. Crawford MLJ, Smith EL III, Harwerth RS, von Noorden GK. Stereoblind monkeys have few binocular neurons. Invest Ophthalmol Vis Sci 1984;25:779-81.

22. Holopigian K, Blake R, Greenwald MJ. Clinical suppression and amblyopia. Invest Ophthalmol Vis Sci 1988;29:444-51.

23. Fahle M. Non-fusible stimuli and the role of binocular inhibition in normal and pathologic vision, especially strabismus. Doc Ophthalmol 1983;55:323-40.

24. von Noorden GK. Binocular vision and ocular motility: theory and management of strabismus. St Louis: CV Mosby, 1990.

25. Smith EL III, Levi DM, Manny RE, Harwerth RS, White JM. The relationship between binocular rivalry and strabismic suppression. Invest Ophthalmol Vis Sci 1985;26:80-7.

26. Richards W. Anomalous stereoscopic depth perception. J Opt Soc Am 1971;61:410-4.

27. Schor C. Zero retinal image disparity: a stimulus for suppression in small angle strabismus. Doc Ophthalmol 1978:46:149-60.

28. McKee SP, Harrad RA. Fusional suppression in normal and stereoanomalous observers. Vision Res 1993;33:1645-58. 